\title{
The Use of Hyaluronic Acid Combined with Teosyal Redensity II for the Treatment of Dark Circles Under the Eyes
}

\author{
VALERIU ARDELEANU1,2, SORIN IOAN BERBECE ${ }^{1 *}$, IOAN PETRE FLORESCU ${ }^{2,3,4}$, RADU CRISTIAN JECAN ${ }^{2,3,5}$ \\ 'Dunarea de J os University of Galati, Faculty of Medicine and Pharmacy, 47 Domneasca Str., 800008, Galati, Romania \\ ${ }^{2}$ Arestetic Clinic, 78 Brailei Str., 800098, Galati, Romania \\ ${ }^{3}$ Carol Davila University of Medicine and Pharmacy, 8 Eroii Sanitari Str., 050474, charest, Romania \\ 4'Bagdasar Arseni Clinical Emergency Hospital, 12 Berceni Road., 041915, Bucharest, Romania \\ ${ }^{5}$ Agripa Ionescu Clinical Emergency Hospital, 7 Ion Mincu Str., 011356, Bucharest, Romania
}

\begin{abstract}
This study intends to highlight the results of the product Teosyal Redensity II through the cumulative effects of its constituents, that have an antioxidant, hydrating, filling and facial restructuring role. The study was performed in the Arestetic Clinic, Galai, between 07.12.2015 and 07.12.2016 on a group of 49 patients, of which 43 women and 6 men. Teosyal Redensity 2 proved to be a safe product - as long as it is injected correctly, it does not cause complications. It's easy to inject and has a very high efficiency. It's a periorbital rejuvenation and restructuring product, with a filling, antioxidant, hydration and dermal redensification effect. In the case of persons that needed a second injection after 1 month, one could observe a major improvement in elasticity and firmness felt by the person performing the injection. The injection requires small quantities of the substance and is accompanied by only mild discomfort. The result is visible right away.
\end{abstract}

Keywords: dark circles, Redensity II, Teosyal, hyaluronic acid, biochemistry

Dark circles represent a rising unaesthetic factor. Dark circles encompass two clinical aspects: the formation of subocular grooves and the brown-blackish coloring of the periocular areas. The appearance of these dark circles is a result of the resorption of the adipose tissue around the eyes, as well as a decrease in the normal arterial irrigation accompanied by the slowing of the venous drainage. Thus, the venous blood stagnates in the capillaries for a longer time, which results in the extravasation of the carboxyhemoglobin and, thus, in the brown-blackish tint of these areas. The Swiss company Teoxane has managed to create a unique formula that can fill the grooves and ensure uniform coloring of this area. Before the appearance of this product, it was attempted to fill the grooves with various products containing hyaluronic acid. However, the suborbital grooves were often replaced by bags under the eyes, as a result of the expansion of the hyaluronic acid and water retention. The innovative chemical formula created by Teoxane resolves these inconveniences, as well as the problem of correcting dark circles. Redensity II contains a unique combination of cross-linked hyaluronic acid and non-cross-linked hyaluronic acid, 8 amino acids, 3 antioxidants, 2 minerals and 1 vitamin. The results are spectacular and last for approximately 1 year.

\section{Experimental part}

\section{Material and method}

The study was performed in the Arestetic Clinic, Galai between 07.12.2015 and 07.12.2016 on a group of 49 patients, on which 43 women and 6 men.

The product used was Teosyal Redensity II, with the following chemical composition: cross-linked hyaluronic acid with BDDE, combined with non-cross-linked hyaluronic acid in a total concentration of $15 \mathrm{mg} / \mathrm{mL}, 8$ amino acids (Leu, Ile, Val, Gly, Lys, Thr, Pro, Arg), 3 antioxidants (Glutathione, N-Acetyl-LCystein, Alpha-Lipoic Acid), vitamin B6 and lidocaine $0.3 \%$. All of these constituents are included in a viscous gel up to $1 \mathrm{ml}$.

The anesthesia was local, through the application of anesthetic cream $9 \%$ lidocaine and 3\% procaine. The injection was performed with a $27 \mathrm{G}, 37 \mathrm{~mm}$ cannula. The injection plane was deep, on the periosteum. The patients were examined after $24 h, 7$ days and 14 days.

The indications were represented by the presence of suborbital grooves and/or the brown-blackish tint. The exclusion criteria were represented by the presence of bags under the eyes, hydrosaline retention and chronic renal failure with eyelid edema.

The clinical evaluation of the patients was realized in accordance with the protocol recommended by the manufacturer, as follows: Stage 1 - pressing down on the inferior orbital edge in order to exclude the presence of lymph stasis. Stage 2 - evaluating the laxity of the lower eyelid skin (Redensity 2 is indicated for $1^{\text {st }}$ degree laxity). Stage 3 - evaluation of the vector of the lower eyelid that should normally be negative. Stage 4 - estimation of the quantity of hyaluronic acid. The patient is asked to look up, while the physician exercises pressure on the zygomatic bone.

\section{Results and discussions}

After turning 30 , more than $50 \%$ of women have dark circles under their eyes. Thus, the treatment of dark circles is the $7^{\text {th }}$ most popular aesthetic treatment (1). After turning 50 , women prefer to treat their lower facial area, while, before turning 45, they prefer to treat their upper facial area (1). This has also been confirmed by our study. The division based on the age group of the patients is displayed in the graph below and is in agreement with other reported studies (2). The ideal substance for treating the periorbital 
area should be biocompatible, non-allergenic, stable, safe and show good aesthetic results; in addition, the result should last for a long period of time, a fact which has also been confirmed by other specialized studies (3). Nowadays, even plastic surgeons increasingly consider the rejuvenation of the periocular area with the help of fillers, searching for the optimum filler that observes the features listed above as closely as possible $(4,5,6)$. This technique is contrasted with the autologous transfer of the patient's own fat (7).

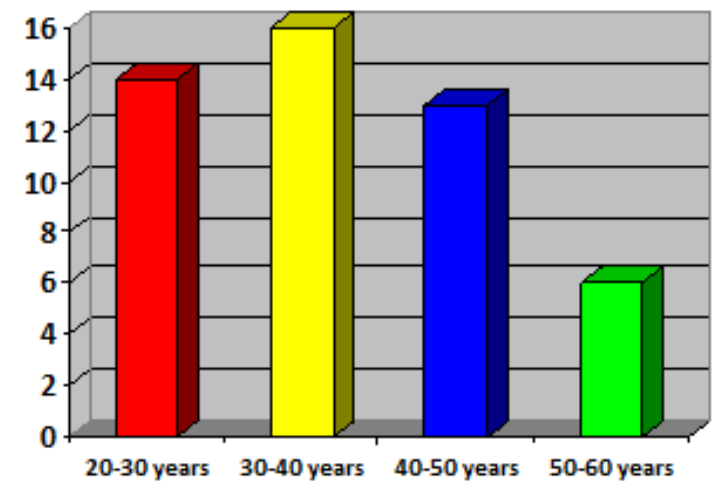

Fig. 1. The distribution of teh patients by age

The quantities used were $1 \mathrm{~mL}$ for 47 patients, $2 \mathrm{~mL}$ for one patient and $3 \mathrm{~mL}$ for a single patient, but the average, generally used quantity was 1 milliliter.

From the point of view of pain, all of the patients could withstand the treatment with local anesthesia and no other adjuvants or anesthesia techniques were necessary. During the procedure, the patients tolerated the operation very well, and only felt a mild discomfort. Due to the anesthetic cream applied before the injection, the sting of the needle was not felt and the lidocaine in the injected product ensured that the patients felt no pain during the injection, or immediately after the injection. We would also like to mention that no patient complained of a numbing of the area in question, which indicates that the product contains the optimum lidocaine dosage.

The result was visible immediately after the injection. The suborbital grooves disappeared in all patients. $10 \mathrm{~min}$ after the injection, the color also started to change and became violet. After the injection, a delicate massage of the injected area was performed along the injection line, using the technique of retrograde injection through the cannula, a technique that is usually also used by other authors (8) (photo 1-4).

At the evaluation performed after 14 days, all of the patients stated that they were very happy with the result and had experienced no complications. 5 patients also

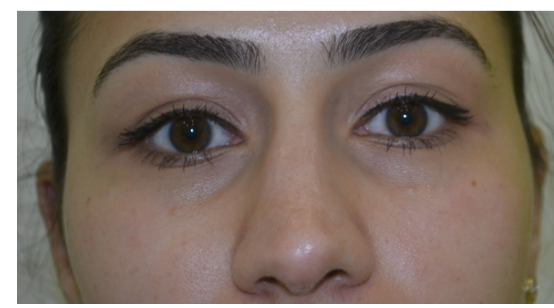

Photo 1. Patient 1: Before injection (1 mL Redensity II)

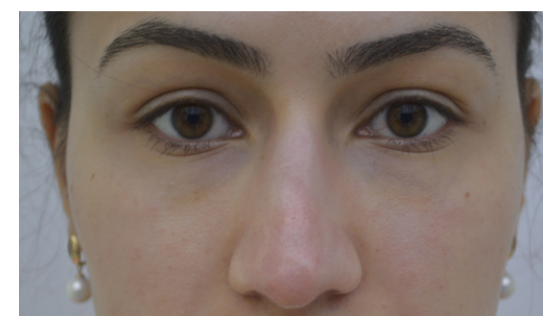

Photo 2. Patient 1:

After 3 day

following

injection ( $1 \mathrm{~mL}$

Redensity II)

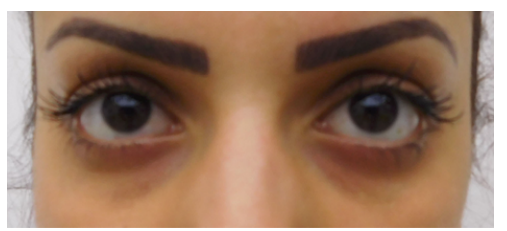

Photo 3. Patient 2: Before injection (1 mL Redensity II)

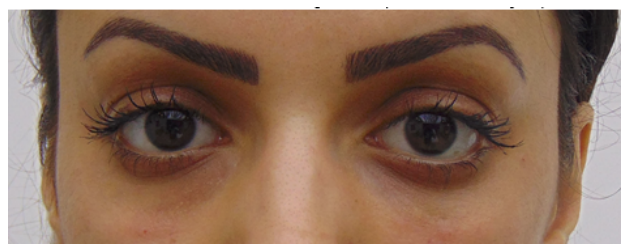

Photo 4. Patient 2: imediatly after injection (1 ml Redensity II)

received injections with botulinic toxin in the upper facial area.

At the evaluation after 1 month, the dark circle area showed normal coloration in 27 patients and a slight violetbluish tint in 6 patients (the one whose dark circles were almost black before the injection). The volumization occurred in all patients, giving the face a more rested and youthful appearance.

14 patients also underwent treatment with botulinic toxin (Vistabel and Azzalure) for the upper facial area, including the area of the external eye canthus (crow's feet). 9 patients were subjected to the treatment 7-8 days before the Redensity injection and 5 patients received botulinic toxin injections 2 weeks after the Redensity treatment. No interactions or adverse reactions between the substances were observed. The two substances did not affect the patients' aesthetic appearance. On the contrary, they improved each other's effect. Given the frequent association between crow's feet and the dynamic w rinkles of the upper facial area, we consider that the combination of Redensityll and botulinic toxin solves the global problem of imperfections in the upper facial area, resulting in a face with a more youthful, bright and rested appearance, with 0 wrinkles and dark circles. There were also 11 patients for which Redensity II was associated with Teosyal Deep lines, with the latter being used to fill nasolabial folds. The injection with Deep Lines was performed 14 days after Redensity II in order to prevent possible water retention in the dark circle area. No cross-reactions or complication were noted in any of these cases either.

With regard to the quantity of injected substance, of the ... patients, $2 \mathrm{ml}$ were used for two patients and $3 \mathrm{ml}$ were used for a single patient (photograph). In the case of the latter, $2 \mathrm{ml}$ were injected in the first session and $1 \mathrm{ml}$ was injected after 14 days. The result was very good compared to the initial appearance, but this was the person that rated the treatment as 9/10. She was happy with the result, but considered that the treatmentwas too expensive. Returning to the subject of the rating, of the 49 patients, 48 rated the result obtained as $10 / 10$ and 1 patient rated it as $9 / 10$, with the average rating being 9.97, and the patients' level of satisfaction being excellent compared to any other studies (9).

Of the total patients, 3 developed a small ecchymosis in the injection spot. It disappeared 5-6 days after the injection. This was the most frequent adverse reaction and cannot be considered a complication (9). Two patients showed a slight morning edema, that lasted for approx. 30 minutes, for 1 week after the injection and disappeared spontaneously.

Specialized literature lists a series of immediate complications/adverse reactions associated with filler injections, such as swelling, erythema, ecchymosis, pain, 
infections, allergic reactions and nodules (10). There are reported cases of post-injection swelling lasting for years in patients consuming alcoholic beverages, after immediate and prolonged exposure to sunlight or after strenuous physical activity performed immediately after the injection (11), which indicates the importance of observing the medical recommendations after the injections. Cutaneous infections associated with the injection of fillers are also documented and are mostly determined by imperfect antisepsis conditions, with the most prominent germ being Staphylococcus epidermidis (12). Post-injection dyschromia, though documented in specialized literature (8) was not recorded in any patient. On the contrary, a uniform discoloration was obtained in the dark circle area and the brown-bluish or blackish color disappeared. In the case of also injecting fillers in the upper eyelid, a heavy eyelid sensation can occur (10). We only used this technique in two patients, of which one needed $2 \mathrm{ml}$ and one needed $3 \mathrm{ml}$, with the entire periocular area being excavated. No patients complained of a heavy eyelid sensation. On the contrary, the two patients said that they felt their upper eyelids were more elastic.

Medium term post-filer complications are listed in the specialized literature as being the appearance of nodules or granuloma, as well as the migration of the substance (10). Since these are also the most difficult to treat, we consider them the most relevant from the point of view of complications and we would like to mention that no patients showed such reactions. However, we w ould also like to note that the specialized literature also states the causes of complications, that are: reactivity of the injection spot, improper injection technique, hypersensitivity to the product, mediated or direct vascular phenomena (10). We would like to highlight the fact that, in order to avoid complications, it's very important to prepare the area correctly before the injection, to obtain information with regard to the patient's allergies and, especially, to use the correct technique (13). In addition, no substance migration and no accelerated resorption were noted.

Due to its formula containing combined hyaluronic acid, Redensity II redensifies the papillary dermis and the dermoepidermal junction, in addition to hydrating the area. According to recent studies at the level of the dermoepidermal junction, an increase in elasticity and firmness was noted - a 98\% increase in the IV collagen and a $26 \%$ increase in fibrin 1 . The collagen is responsible for maintaining tissue elasticity and cohesion and the fibrin has a tensor effect on the skin; due to the three antioxidants, one could observe a $28 \%$ decrease in malondialdehyde, the main marker of oxidative stress; the hydration of the skin was evaluated in accordance with the studies by measuring x14 epidermal glycosaminoglycan, the production of which increased considerably after the injection $(14,15)$.

We believe that Redensity II shows excellent results when injected correctly, with no complications. In most cases, $1 \mathrm{~mL}$ of the substance is sufficient. However, when injected incorrectly (superficially), small edemas or bags could appear and last for a long time. Injected into the muscle, like any filler containing hyaluronic acid, itshall be dislocated very quickly, which shall lead to an accelerated resorption and the disappearance of the desired effect in a short time (16). Before using Redensity II, we used other products containing hyaluronic acid for injections in the dark circle area. These had a low molecular density, but the patients frequently developed small bags under the eyes, that could last for several months. Redensity ll seems to have solved this problem due to the combined use of cross-linked and non-cross-linked hyaluronic acid, as well as due to the use of a gel with low hygroscopic properties, with a low consistency and a viscosity with optimized cohesion, but which also has a dermal stimulation capacity $(17,18)$.

From the point of view of durability, this study was performed for a period of one calendar year, with its purpose being to highlight the advantages of Redensity II and the almost inexistent risk of complications. How ever, we would like to mention that we've been using this product for approx. 3 years and the average remanence time is approx. 14-16 months, which is even superior to the injection of classic fillers in this area, that have a higher risk of complications (9).

\section{Conclusions}

Teosyal Redensity 2 is a safe product, that does not determine complications when injected correctly and only causes rare and minimal adverse reactions: erythema, edema, ecchymosis, discomfort. It's easy to inject and has a very good efficiency, with a patient satisfaction level of $98 \%$. It's not like other filling products with hyaluronic acid, it's a periorbital rejuvenation and restructuring product, with the following effects: filling, antioxidant, hydration, dermal redensification, IV collagen and fibrin production increase, with an increase in skin elasticity and firmness. In the case of persons who needed a second injection after 1 month, one could observe a much better elasticity and firmness felt by the person performing the injection. The injection requires small quantities of substance and is accompanied by a minimum discomfort. The result is immediately visible.

\section{References}

1. NARURKAR, V., SHAMBAN, A., SISSINS, P., STONEHOUSE, A., GALLAGHER, C. Facial treatment preferences in aesthetically aware women. Dermatol Surg. 2015, 41 Suppl 1:S153-60

2. MORLEY, A.M. AND R. MALHOTRA, Use of hyaluronic acid filler for tear-trough rejuvenation as an alternative to lower eyelid surgery. Ophtal Plast Reconstr Surg, 2011. 27(2): p. 69-73

3. BRAVO, BS., ROCHA, CR., BASTOS, JT., SILVA PM., Comprehensive treatment of periorbital region with hyaluronic acid. J Clin Aesthet Dermatol. 2015, 8(6): 30-5

4. LIEW S, NGUYEN DQ. Nonsurgical volumetric upper periorbital rejuvenation: a plastic surgeon's perspective. Aesth Plast Surg. 2011; 35:319-325;

5. MORAL ML. Office-based periorbital rejuvenation. Facial Plast Surg. 2013; 29:58-63;

6. HOENING, J., Hoening D. Minimally invasive periorbital rejuvenation. Facial Plast Surg. 2013; 29:295-309

7. KRANEDONK S., OBAGI S. Autologous fat transfer for periorbital rejuvenation: indications, technique and complications. Dermatol Surg. 2007; 35:572-578

8. BRUNA SOUZA FELIX BRAVO, CAMILA ROOS MARIANO DA ROCHA, JULIEN TONI DE BASTOS AND PRISCILA MARA CHAVES E SILVA. Comprehensive treatment of periorbital region with hyaluronic acid. J Clin Aesthet Dermatol. 2015 J un; 8 (6): 30-35

9. GOLDBERG RA, FIASCHETTI D. Filling the periorbital hollows with hyaluronic acid gel: initial experience with 244 injections. Ophthal PlastReconst Surg. 2006;22:335-341-341.341-343

10. PHILIPPE LAFAILLE, ANTHONY BENEDETTO. Fillers: Contraindications, side effects and precautions. J Cutan Aesthet Surg. 2010 Jan-Apr; 3(1): 16-19.

11. DUFFY DM. Complications of fillers: Overview. Dermatol Surg. 2005; 31:1626-33

12. CHRISTENSEN L. Normal and pathologic tissue reactions to soft tissue gel fillers. Dermatol. Surg 2007; 33:S168-75

13. GOLD M. The science $\&$ art of hyaluronic acid dermal filler use in esthetic applications. J Cosmet Dermatol. 2009; 8:301-7 
14. *** Study report 10E2047 TEOSYAL $\circledast$ PureSense Redensity [II] on human skin explants carried out by BIO-EC for Teoxane, 2010 15.*** Study report 13E2855, Evaluation of the diffusion of injectable products on human skin explants ex vivo, carried out by BIO-EC for Teoxane, 2014

16. LEMPERLE G, RULLAN PP, GAUTHIER-HAZAN N. Avoiding and treating dermal filler complications. Plast Reconstr Surg. 2006; 118:S92107
17. MICHAUD, T., GASSIA, V., BELHAOUARI, L. Facial dynamics and emotional expressions in facial aging treatments. J Cosmet dermatol. 2015, 14(1):9-21;

18. BASS, L. Injectable filler techniques for facial rejuvenation, volumization and augmentation. Facial Plast Surg Clin N Am. 2015, 23:479-488

Manuscript received: 3.12 .2016 